\title{
CONSTITUINTES QUÍMICOS E ATIVIDADE ANTIMICROBIANA DOS EXTRATOS DE Dilodendron bipinnatum
} (SAPINDACEAE) $)^{\#}$

\author{
Josiane Cristina dos Santos, Carlos Alberto Nastally de Oliveira, Larissa Varella e Andréia Pereira Matos \\ Faculdade de Farmácia, Centro Universitário Central Paulista, 13563-470 São Carlos - SP, Brasil \\ Ana Paula Terezan, Ana Cristina Leite, João Batista Fernandes*, Paulo Cezar Vieira e Maria Fátima das Graças \\ Fernandes da Silva \\ Departamento de Química, Universidade Federal de São Carlos, CP 676, 13565-905 São Carlos-SP, Brasil \\ José Rubens Pirani \\ Departamento de Botânica, Instituto de Biociências, Universidade de São Paulo, CP 11461, 05508-900 São Paulo-SP, Brasil
}

Recebido em 16/6/10; aceito em 1/10/10; publicado na web 27/10/10

\begin{abstract}
CHEMICAL CONSTITUENTS AND ANTIMICROBIAL ACTIVITY OF EXTRACTS FROM Dilodendron bipinnatum (SAPINDACEAE). The phytochemical investigation of ethanolic extracts from leaves, branches and stems of D. bipinnatum afforded the steroids $\beta$-sitosterol, stigmasterol, campesterol, sitostenone and sitosterol-3-O- -D-glycopyranoside, along with two cycloartane triterpenes: cycloeucalenol and 24-methylenecycloartenol. The antimicrobial activity of the extracts was evaluated against Staphylococcus aureus (ATCC 25923), Escherichia coli (ATCC 25922), Bacillus subtilis (ATCC 6623), Pseudomonas aeruginosa (ATCC 15442), Micrococcus luteus (ATCC 9341) and Candida albicans (ATCC 10231). The extracts of the leaves and branches showed moderate activity against Candida albicans. The extract of the branches was active against Micrococcus luteus. This is the first report on the phytochemical study of D. bipinnatum.
\end{abstract}

Keywords: Sapindaceae; Dilodendron bipinnatum; antimicrobial activities.

\section{INTRODUÇÃO}

O gênero Dilodendron é constituído por apenas três espécies: $D$. bipinnatum, D. costaricense e D. elegans, sendo que a primeira tem ocorrência restrita a regiões secas do Brasil. ${ }^{1}$ Espécies da família Sapindaceae têm sido usadas na medicina tradicional como diuréticas, estimulantes, expectorantes, sedativas, vermífugas e no tratamento de dermatites. ${ }^{2}$ Saponinas, sesquiterpenos acíclicos e diterpenos oligoglicosídicos são os principais metabólitos secundários presentes em diversas espécies de Sapindaceae usadas na medicina oriental. ${ }^{3}$ Das atividades biológicas descritas para espécies de Sapindaceae, destaca-se a atividade antimicrobiana. ${ }^{4} \mathrm{Um}$ dos primeiros relatos sobre o potencial antimicrobiano das sapindáceas foi descrito por Lemos et al., ${ }^{5}$ que avaliaram a atividade da saponina hederagenina $3-O-\beta-[\alpha-L-r a m n o p i r a n o s i l-(1 \rightarrow 3)-\beta-D-$ glicopiranosídeo], isolada de Sapindus saponaria, sobre os microorganismos Pseudomonas aeruginosa, Bacillus subtilis e Cryptococcus neoformans. Além disso, quatro saponinas triterpênicas foram isoladas do extrato metanólico do pericarpo de Sapindus rarak que apresentou atividade moluscicida. ${ }^{6}$ Um estudo semelhante mostrou a atividade de extratos brutos de $S$. saponaria e de duas saponinas frente a várias espécies de Candida isoladas da secreção vaginal de pacientes que sofriam de candidíase vulvovaginal. ${ }^{3}$ Recentemente, foi relatada a atividade antimicrobiana de várias espécies de Sapindaceae, como Hippobromus pauciflorus, ${ }^{7}$ Lecaniodiscus cupanoides ${ }^{8}$ e Dodonaea viscosa. ${ }^{9}$

O presente trabalho descreve o isolamento e caracterização estrutural dos esteroides $\beta$-sitosterol, estigmasterol, campesterol, sistotenona e sitosterol 3-O- $\beta$-D-glicopiranosídeo e dos triterpenos cicloartânicos: cicloeucalenol (1) e 24-metilenocicloartanol (2). As atividades antimicrobianas dos extratos de folhas, galhos e caule de D. bipinnatum e triterpenos foram avaliadas.

*e-mail: djbf@power.ufscar.br

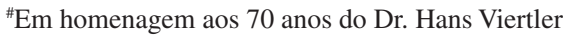

\section{PARTE EXPERIMENTAL}

\section{Procedimentos experimentais gerais}

Os espectros de RMN foram obtidos em espectrômetro Bruker DRX 400, observando ${ }^{1} \mathrm{Ha} 400 \mathrm{MHz}^{13} \mathrm{C}$ a $100 \mathrm{MHz}$; utilizou-se $\mathrm{CDCl}_{3}$ como solvente e TMS como padrão interno. Os espectros de massas foram obtidos em aparelho da Micromass, modelo Micromass Quattro LC.

Para identificação dos esteroides foi utilizado cromatógrafo a gás acoplado ao espectrômetro de massas, marca Shimadzu, modelo QP5000, com coluna capilar DB-5 (30 m x 0,25 mm), utilizando as seguintes condições: temperatura do injetor: $250^{\circ} \mathrm{C}$, gás de arraste: $\mathrm{He}$, temperatura inicial do forno: $150{ }^{\circ} \mathrm{C}$ por $1 \mathrm{~min}$, velocidade de aquecimento a $6{ }^{\circ} \mathrm{C} / \mathrm{min}$ até $280^{\circ} \mathrm{C}$, permanecendo nessa temperatura por $15 \mathrm{~min}$. Os espectros de massas foram obtidos por impacto de elétrons a $70 \mathrm{eV}$.

As separações cromatográficas em colunas foram realizadas utilizando-se gel de sílica 230-400 mesh. As separações por cromatografia líquida de alta eficiência (CLAE), em condições preparativas, foram realizadas em coluna Asahipak GS-310 2G (2,15 x 50,0 cm), utilizando-se equipamento Shimadzu LC-8A com válvula de reciclo, injetor Rheodyne 7123 com loop $500 \mu \mathrm{L}$ e detector UV/VIS Shimadzu SPD-6AV.

As análises cromatográficas em camada fina foram realizadas em cromatoplacas de gel de sílica $\mathrm{F}_{254}$ sobre placa de alumínio Merck, de 0,2 mm de espessura, empregando-se uma solução de vanilina/ácido sulfúrico como revelador.

\section{Material vegetal}

As folhas, galhos e caules de D. bipinnatum Radlk foram coletados e identificados pelo Prof. Dr. J. R. Pirani, do Departamento de Botânica, Instituto de Biociências da USP, no dia 21/01/2001 na cidade de Bom Jesus do Goiás (GO). A exsicata da espécie encontra-se depositada no herbário do IB-USP, sob o número 4764. 


\section{Preparação dos extratos}

As folhas (1482,2 g), galhos (543,7 g) e caules $(1262,2 \mathrm{~g})$ de $D$. bipinnatum foram secos em estufa de circulação de ar a $40{ }^{\circ} \mathrm{C}$, por aproximadamente $48 \mathrm{~h}$ e moídos em liquidificador. As partes vegetais da planta foram submetidas separadamente à extração com etanol por 7 dias, em temperatura ambiente e em repouso. Após este período, o solvente foi evaporado em evaporadores rotativos, obtendo-se os respectivos extratos brutos.

\section{Isolamento das substâncias de $D$. bipinnatum}

O extrato etanólico dos galhos de $D$. bipinnatum $(4,1 \mathrm{~g})$ foi submetido à cromatografia em coluna ( $28 \mathrm{~cm}$ de diâmetro x 17,3 $\mathrm{cm}$ de comprimento, $390 \mathrm{~g}$ de gel de sílica), utilizando-se hexano, hexano/AcOEt 9:1, hexano/AcOEt 1:1, AcOEt e MeOH em eluição gradiente (700 mL cada fração), levando a seis sub-frações: Dbge1

Dbge6. A sub-fração Dbge3 $(21,0 \mathrm{mg})$ foi submetida a fracionamento em coluna de gel de sílica $(28 \mathrm{~g}, 2 \mathrm{~cm}$ de diâmetro x $35 \mathrm{~cm}$ de comprimento) com eluição gradiente, utilizando-se hexano/AcOEt (10 a 100\%) e $\mathrm{MeOH}(100 \%)$ (14 frações de $10 \mathrm{~mL}$ ), fornecendo a mistura de $\beta$-sitosterol, estigmasterol, campesterol e sistotenona (sub-fração Dbge 3.8, 5,2 mg).

O extrato etanólico das folhas de D. bipinnatum $(12,3 \mathrm{~g})$ foi fracionado através de cromatografia em coluna de gel de sílica (500 g, $17,2 \mathrm{~cm}$ de diâmetro x $35 \mathrm{~cm}$ de comprimento) utilizando-se hexano/ AcOEt (10 a 100\%) e MeOH (100\%) como eluentes, obtendo-se 38 sub-frações (10 mL cada): Dbfe1 a Dbfe38. Utilizando-se estas mesmas condições cromatográficas, os fracionamentos das sub-frações Dbfe5 $(82,5 \mathrm{mg})$ e Dbfe6 (129,6 mg) levaram ao isolamento de $\mathbf{1}(7,0 \mathrm{mg})$ e do sitosterol 3-O- $\beta$-D-glicopiranosídeo $(6,5 \mathrm{mg})$, respectivamente.

Visando o isolamento de substâncias em maiores quantidades, uma nova porção de extrato etanólico de folhas de $D$. bipinnatum $(11,1 \mathrm{~g})$ foi fracionada, utilizando-se os mesmos parâmetros aplicados no fracionamento anterior, obtendo-se 162 sub-frações (Dbfe1-1 a Dbfe1-162). A sub-fração Dbfe1-29 (102,3 mg) foi submetida a fracionamento através de cromatografia em coluna de gel de sílica utilizando-se hexano/AcOEt (10-100\%) e MeOH (100\%) como eluentes, obtendo-se 12 sub-frações de 10 mL: Dbfe1-29-1 a Dbfe1-29-12. A sub-fração Dbfe1-29-12 (9,7 $\mathrm{mg}$ ) foi submetida à CLAE preparativa com hexano/AcOEt $(85: 15)$ como eluente, levando ao isolamento da substância 2 (2,8 mg).

\section{Ensaios antimicrobianos}

Os ensaios antimicrobianos foram realizados utilizando-se a técnica de microdiluição em caldo proposta pelo National Commite for Clinical Laboratory Standards (NCCLS) M7-A6 ${ }^{10}$ e adaptação do M27-A2, ${ }^{11}$ para determinação do CIM (menor concentração capaz de inibir o crescimento do micro-organismo avaliado). As determinações de CIM foram realizadas em triplicata em microplacas com 96 poços. Os micro-organismos utilizados da American Type Culture Collection foram Staphylococcus aureus (ATCC 25923), Escherichia coli (ATCC 25922), Bacillus subtilis (ATCC 6623), Pseudomonas aeruginosa (ATCC 15442), Micrococcus luteus (ATCC 9341) e Candida albicans (ATCC 10231). As soluções-estoque das amostras testadas foram preparadas em frasco tipo Eppendorf, solubilizando-se $1 \mathrm{mg}$ de amostra em $40 \mu \mathrm{L}$ de DMSO. Essas soluções foram diluídas em $960 \mu \mathrm{L}$ do caldo Mueller-Hinton para bactérias ou caldo Sabouraud para o ensaio com a levedura. A partir dessa solução as concentrações finais variaram de 7,81 a $500 \mu \mathrm{g} / \mathrm{mL}$. Os inóculos foram padronizados utilizando-se a escala de 0,5 de Mc Farland de turbidez padrão $\left(10^{8} \mathrm{UFC} / \mathrm{mL}\right)$ e diluídos na razão 1:10 no caldo para o procedimento de microdiluição. Após a micropipetagem, as microplacas foram tampadas e incubadas a $37^{\circ} \mathrm{C}$ por 18-24 h sem agitação. Terminado o período de incubação, os resultados foram visualizados e os poços que não apresentaram crescimento aparente foram selecionados para determinar a atividade microbicida (CBM ou CFM) ou microbiostática das amostras. Essa determinação foi realizada através de subculturas em placas de Petri utilizando-se ágar Mueller-Hinton para o crescimento de bactérias e ágar Sabouraud para o crescimento do fungo. As placas de Petri foram incubadas a 37 ${ }^{\circ} \mathrm{C}$ por $48 \mathrm{~h}$ e verificou-se a presença/ausência de colônias microbianas. Após o preparo das subculturas foram adicionados em cada orifício das placas $15 \mu \mathrm{L}$ de resazurina a $0,01 \%$ em solução aquosa esterilizada onde, após $4 \mathrm{~h}$ de reincubação, a leitura foi realizada. ${ }^{12}$

Dessa maneira foi possível determinar a menor concentração de cada extrato capaz de inibir o crescimento dos micro-organismos através de indicadores diluídos na solução. Todos os ensaios foram realizados com controle negativo (DMSO), controle positivo (cloridrato de tetraciclina, cloridrato de vancomicina e nistatina), controle do crescimento do micro-organismo e controle de precipitação da amostra, evitando possibilidade de resultado falso-negativo ou falso-positivo.

\section{RESULTADOS E DISCUSSÃO}

O estudo químico do extrato etanólico de galhos e folhas de $D$. bipinnatum resultou na identificação dos esteroides $\beta$-sitosterol, estigmasterol, campesterol, sistotenona (em mistura) ${ }^{13}$ e sitosterol ${ }^{14}$ e dos triterpenos: cicloeucalenol (1) ${ }^{15,16}$ e 24-metilenocicloartanol $(\mathbf{2})^{15}$ (Figura 1). Estes compostos foram caracterizados através de experimentos de $\mathrm{RMN}{ }^{1} \mathrm{H} \mathrm{e}{ }^{13} \mathrm{C}$ e pela comparação com os respectivos dados relatados na literatura. A mistura dos esteroides foi também caracterizada via CG-EM. Este é o primeiro relato de metabólitos secundários isolados de $D$. bipinnatum.

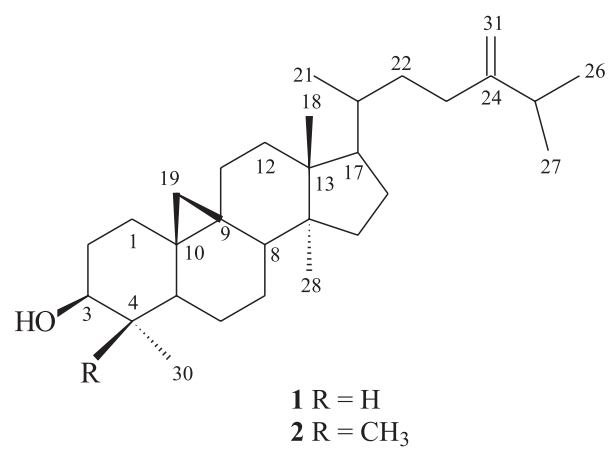

Figura 1

A mistura dos esteroides foi identificada através de análises por CG-EM, comparando-se os espectros de massas obtidos com os espectros existentes no banco de dados do equipamento, por RMN ${ }^{1} \mathrm{H}$ e ${ }^{13} \mathrm{C}$ e também por comparação com dados da literatura. ${ }^{13} \mathrm{O}$ cromatograma de íons totais da análise por CG-EM apresentou 4 picos. Os espectros de massas dos picos apresentaram íons moleculares $\mathrm{m} / \mathrm{z}, 414,412,400$ e 412 e fragmentos concordantes com aqueles de sitosterol, estigmasterol, campesterol e sitostenona, respectivamente.

Os extratos de folhas (Dbfe), galhos (Dbge) e caule (Dbce) de $D$. bipinnatum foram submetidos aos ensaios antimicrobianos (Tabela 1), obtendo-se os dados de concentração inibitória mínima (CIM), concentração bactericida mínima (CBM) e concentração fungicida mínima (CFM). Os resultados foram analisados seguindo o modelo proposto por Aligianis et al.,${ }^{17}$ que classifica os materiais vegetais de acordo com a CIM apresentada, sendo considerada como forte inibição $500 \mu \mathrm{g} / \mathrm{mL}$, moderada 600 a $1500 \mu \mathrm{g} / \mathrm{mL}$ e fraca acima de $1600 \mu \mathrm{g} / \mathrm{mL}$. Os extratos etanólicos dos galhos (Dbge) e das folhas (Dbfe) apresentaram a CIM igual a $250 \mu \mathrm{g} / \mathrm{mL}$ e CFM igual a $500 \mu \mathrm{g} /$ 
Tabela 1. Atividade antimicrobiana dos extratos etanólicos de Dilodendron bipinnatum e de cicloeucalenol e 24-metilenocicloartenol

\begin{tabular}{|c|c|c|c|c|c|c|c|c|}
\hline \multirow{3}{*}{ Amostras } & \multicolumn{8}{|c|}{ Concentração Mínima ( $\mu \mathrm{g} / \mathrm{mL})$} \\
\hline & \multirow{2}{*}{$\begin{array}{c}\text { S. aureus } \\
\text { CIM }\end{array}$} & \multirow{2}{*}{$\begin{array}{c}\text { E. coli } \\
\text { CIM }\end{array}$} & \multirow{2}{*}{$\begin{array}{c}\text { B. subtilis } \\
\text { CIM }\end{array}$} & \multirow{2}{*}{$\begin{array}{l}\text { P. aeruginosa } \\
\text { CIM }\end{array}$} & \multicolumn{2}{|c|}{ M. luteus } & \multicolumn{2}{|c|}{ C. albicans } \\
\hline & & & & & CIM & $\mathrm{CBM}$ & CIM & CFM \\
\hline Dbfe & $*$ & $*$ & $*$ & $*$ & $*$ & $*$ & 250 & 500 \\
\hline Dbge & $*$ & $*$ & $*$ & $*$ & 250 & 500 & 250 & 500 \\
\hline Dbce & $*$ & $*$ & $*$ & $*$ & $*$ & $*$ & $*$ & $*$ \\
\hline Cicloeucalenol & $*$ & $*$ & $*$ & $*$ & $*$ & $*$ & $*$ & $*$ \\
\hline $\begin{array}{l}\text { 24-metileno } \\
\text { cicloartenol }\end{array}$ & $*$ & $*$ & $*$ & $*$ & $*$ & $*$ & $*$ & $*$ \\
\hline \multicolumn{9}{|l|}{ Controle positivo } \\
\hline $\mathrm{CT}$ & 0,75 & 3,125 & & 1,5625 & 0,1875 & & & \\
\hline $\mathrm{N}$ & & & & & & & 6,25 & \\
\hline $\mathrm{CV}$ & & & 0,0046 & & & & & \\
\hline
\end{tabular}

(*) sem atividade nas concentrações entre o intervalo de 7,81 a $500 \mu \mathrm{g} / \mathrm{mL}$. CT: Cloridrato de tetraciclina; N: Nistatina; CV: Cloridrato de vancomicina; CIM: Concentração Inibitória Mínima; CBM: Concentração Bactericida Mínima; CFM: Concentração Fungicida Mínima

$\mathrm{mL}$ sobre $C$. albicans. Na bactéria Gram-positiva M. luteus o extrato etanólico dos galhos apresentou a CIM igual a $250 \mu \mathrm{g} / \mathrm{mL}$ e CBM igual a $500 \mu \mathrm{g} / \mathrm{mL}$. O extrato etanólico das folhas (Dbfe) não apresentou nenhuma atividade sobre as bactérias. O extrato etanólico dos caules e os triterpenos cicloartanos ( 1 e 2 ) não apresentaram atividade em nenhum dos organismos testados (Tabela 1). As atividades antimicrobianas dos extratos de folhas e galhos podem estar relacionadas à alta quantidade de compostos lipofílicos presentes nos extratos, visto que a lipofilicidade está intimamente ligada à permeação através da camada lipídica dos micro-organismos. ${ }^{18}$

Considerando-se que os extratos de plantas geralmente possuem composições muito complexas e que as substâncias ativas podem estar presentes em concentrações muitos pequenas, aquele de galhos (Dbge), segundo o modelo proposto por Aligianis et al. ${ }^{17}$ apresentou uma ótima atividade sobre C. albicans e sobre a bactéria M. luteus. Na literatura não são encontrados relatados de atividade antimicrobiana significativa para os compostos identificados nos extratos estudados. Sitosterol, que está presente no extrato dos galhos de D. bipinnatum, segundo Sanches et al. ${ }^{19}$ apresentou atividade sobre $S$. aureus com CIM de $100 \mu \mathrm{g} / \mathrm{mL}$, valor considerado pouco ativo para substâncias puras. Estes resultados sugerem novos estudos em modelos animais in vitro, visando conhecer a eficácia e toxicidade, e a utilização dos extratos Dbge e Dbfe como bactericida e/ou fungicida, assim como caracterizar os compostos responsáveis pelas atividades.

\section{MATERIAL SUPLEMENTAR}

Disponível em http://quimicanova.sbq.org.br, na forma de arquivo PDF.

\section{AGRADECIMENTOS}

Ao CNPq, CAPES, Instituto Nacional de Ciência e Tecnologia de Controle Biorracional de Insetos Pragas e FAPESP, pelas bolsas e apoios financeiros.

\section{REFERÊNCIAS}

1. Gentry, A. H.; Steyermark, J.; Annals Ann. Mo. Bot. Gard. 1987, 74, 533.

2. Hegnauer, W.; Chemotaxonomie der Pflanzen, Sapindaceae, Springer Verlag: Berlin, 1970; Martinez, M.; Las Plantas Medicinales de Mexico, Editorial Botas: México, 1992; Sastri, B. N.; The Wealth of India, Raw Materials, CSIR: New Delhi, 1962; Kimata, H.; Nakashima, T.;
Kokubun, S.; Nakayama, K.; Mitoma, Y.; Kitahara, T.; Yata, N.; Tanaka, O.; Chem. Pharm. Bull. 1983, 31, 1998.

3. Tsuzuki, J. K.; Svidzinski, T. I. E.; Shinobu, C. S.; Silva, L. F. A.; Rodrigues-Filho, E.; Cortez, D. A. G.; Ferreira, I. C. P.; An. Acad. Bras. Ciênc. 2007, 79, 577.

4. Pendota, S. C.; Grierson, D. S.; Afolayan, A. J.; Pharm. Biol. 2009, 47, 309; Khurram, M.; Khan, M. A.; Hameed, A.; Abbas, N.; Qayum, A.; Inayat, H.; Molecules 2009, 14, 1332; Pelegrini, D. D.; Tsuzuki, J. K.; Amado, C. A. B.; Cortez, D. A. G.; Ferreira, I. C. P.; Lat. Am. J. Pharm. 2008, 27, 922.

5. Lemos, T. L. G.; Mendes, A. L.; Sousa, M. P.; Braz-Filho, R.; Fitoterapia 1992, 63, 515.

6. Braz Filho, R.; Quim. Nova 2010, 33, 229.

7. Pendota, S. C.; Grierson, D. S.; Afolayan, A. J.; Pharm. Biol. 2009, 47, 309.

8. Oboh, I. E.; Obasuyi, O.; Akerele, J. O.; Acta Pol. Pharm. 2008, 65, 565.

9. Teffo, L. S.; Aderogba, M. A.; Eloff, J. N.; S. Afr. J. Bot. 2010, 76, 25.

10. National Committee for Clinical Laboratory Standard; Methods for Dilution Antimicrobial Susceptibility Tests for Bacteria That Grow Aerobically, Approved Standard - M7-A6, $6^{\text {th }}$ ed., NCCLS/CLSI: Wayne, 2003, vol. 23.

11. National Committee for Clinical Laboratory Standard; Método de Referência para Testes de Diluição em Caldo para a Determinação da Sensibilidade a Terapia Antifúngica das Leveduras, $2^{\text {nd }}$ ed., Norma Aprovada (M27-A2), NCCLS/CLSI: Wayne, 2002.

12. Alves, G. E.; Vinholis, A. H. C.; Casemiro,L. A.; Furtado, N. A. J. C.; Andrade e Silva, M. L.; Cunha, W. R.; Martins, C. H. G.; Quim. Nova 2008, 31, 1226.

13. Müller, A. H.; Vieira, P. C.; Silva, M. F. G. F. da; Fernandes, J. B.; Phytochemistry 1995, 40, 1797.

14. Matida, A. K.; Rossi, M.; Almeida Blumenthal, E. E.; Schuquel, I. T. A.; Malheiros, A.; Vidotti, G. J.; An. Ass. Brasil. Quim. 1996, 45, 147.

15. Kawai, S.; Takada, Y.; Tsuchida, S.; Kado, R.; Kimura, J.; Fish. Sci. 2007, 73, 902.

16. Chang, C. W. J.; Flament, I.; Matson, J. A.; Nishida, T.; Ohloff, G.; Wehrli, F. W.; Weinheimer, A. J.; Progress in the Chemistry of Organic Natural Products, Wien Spring-Verlag: New York, 1979, vol. 36, p. 93.

17. Aligiannis, N.; Kalpoutzakis, E.; Mitaku, S.; Chinou, I. B.; J. Agric. Food Chem. 2001, 49, 4168.

18. Tokuyama, R.; Takahashi, Y.; Tomita, Y.; Tsubouchi, M.; Yoshida, T.; Iwasaky, N.; Kado, N.; Okezaki, E.; Nagata, O.; Chem. Pharm. Bull. 2001, 49, 353.

19. Sanches, N. R.; Cortez, D. A. G.; Schiavini, M. S.; Nakamura, C. V.; Dias-Filho, B. P.; Braz. Arch. Biol. Technol. 2005, 48, 429. 